\title{
Overshadowing of explicitly unpaired conditioned inhibition is disrupted by preexposure to the overshadowed inhibitor
}

\author{
AARON P. BLAISDELL, HERNÁN I. SAVASTANO, and RALPH R. MILLER \\ State University of New York, Binghamton, New York
}

\begin{abstract}
In two conditioned lick-suppression experiments with rats, the interaction of preexposure to a target stimulus and subsequent overshadowing of conditioned inhibition treatment was examined. Blaisdell, Bristol, Gunther, and Miller (1998) have demonstrated that stimulus preexposure and overshadowing counteract each other in their effects on Pavlovian excitation, producing strong excitatory responding to the target stimulus. In the present experiments, a conditioned inhibition analogue of this effect was examined. Overshadowing of conditioned inhibition (i.e., attenuation of inhibitory control of behavior by the target stimulus) was demonstrated when a more salient stimulus was compounded with the target inhibitor during conditioned inhibition training. However, the overshadowing deficit was attenuated by preexposing the target stimulus prior to overshadowing treatment. To account for this phenomenon, we contrast the comparator hypothesis (Miller \& Matzel, 1988) with contemporary models that focus on associative acquisition.
\end{abstract}

In the study of Pavlovian conditioning, preexposure to a conditioned stimulus (CS) and overshadowing are two treatments that are known to attenuate excitatory conditioned responding. However, Blaisdell, Bristol, Gunther, and Miller (1998) recently showed that the compound effect of these treatments is not to summate their deleterious effects on conditioned responding. Instead, CS preexposure and overshadowing counteracted each other, resulting in a strong conditioned response. Toward illuminating the processes underlying this counterintuitive observation, in the present research, we investigated whether CS preexposure and overshadowing treatment would also counteract each other in attenuating conditioned inhibitory control of behavior by a CS.

CS preexposure treatment consists of extended nonreinforced exposure to a CS prior to pairing that CS with an unconditioned stimulus (US). Typically, behavioral control by that $\mathrm{CS}$ is retarded, relative to a nonpreexposed control. The CS preexposure effect is also known as latent inhibition (Lubow, 1973; Lubow \& Moore, 1959), because the effect was originally thought to result from conditioned inhibition acquired by the CS during preexposure, which impaired subsequent acquisition of an excitatory association with the US. However, later research demonstrated

Support for this research was provided by NIMH Grant 33881. We thank Francisco Arcediano, Daniel Burger, James Denniston, Martha Escobar, and Russell Wishtart for comments on a preliminary draft of this manuscript. Thanks are also due Martha Escobar, James Esposito, Gibbi George, and Russell Wishtart for assistance with the collection of data. Correspondence concerning this article should be addressed to R. R. Miller, Department of Psychology, SUNY-Binghamton, Binghamton, NY 13902-6000 (e-mail: rmiller@binghamton.edu).

Note-Vincent M. LoLordo served as action editor for this paper. that the phenomenon was not a form of conditioned inhibition (Rescorla, 1971). Currently, the CS preexposure effect is viewed by many as resulting from a loss of attention or associability to the preexposed CS as a result of the nonreinforced presentations (Lubow, Schnur, \& Rifkin, 1976; Lubow, Weiner, \& Schnur, 1981; Mackintosh, 1975; Pearce \& Hall, 1980).

More recent models provide nonattentional accounts of the CS preexposure effect. For example, the comparator hypothesis (Miller \& Matzel, 1988; Miller \& Schachtman, 1985 ) is a performance rule that states that responding to a CS is determined by the associative strength of the target CS-US association, relative to the associative strength to the US of the stimulus that has the strongest withincompound association with the target CS (excluding the US) that was present during training. This stimulus is hereafter called the target CS's comparator stimulus. In this framework (illustrated in Figure 1), presentation of the target CS at test directly activates a representation of the US as a result of the target CS-US association (link 1). This direct US representation is then compared with the US representation indirectly activated by the target CS-comparator stimulus association (link 2) and the comparator stimulus-US association (link 3 ). The degree of excitatory responding to the CS is directly related to the activation of the direct US representation and inversely related to the activation of the indirect US representation. Conversely, the degree of inhibitory responding to the CS is directly related to the activation of the indirect US representation and inversely related to the activation of the direct US representation. The CS preexposure effect is viewed as being the result of the context having been established as the comparator stimulus for the $\mathrm{CS}$, as a consequence of the strong $\mathrm{CS}-$ context 


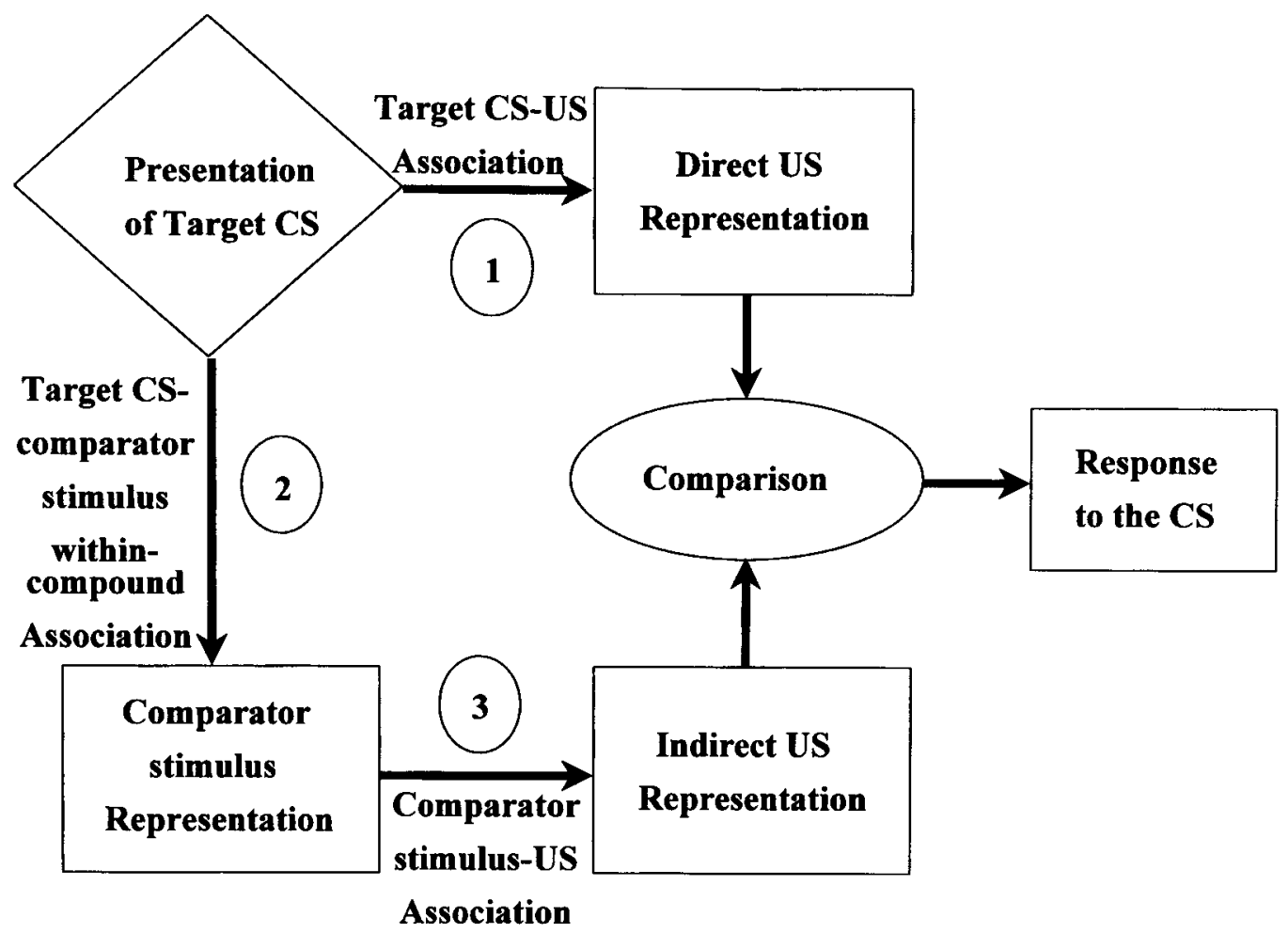

Figure 1. The comparator hypothesis. CS, conditioned stimulus; US, unconditioned stimulus.

within-compound association that is formed during the preexposure treatment (Grahame, Barnet, Gunther, \& Miller, 1994). Subsequent CS-US pairings establish a moderate CS-US association, as well as a moderate context-US association (moderate, because there are typically only a few retardation test pairings). However, expression of the CS-US association (as evidenced by responding to the $\mathrm{CS}$ ) is retarded (i.e., latent inhibition) owing to the strong CS-context association (link 2) that was acquired during the preexposure phase (in conjunction with the context-US association [link 3] that was acquired during training with the US). In contrast, when no preexposure to the target stimulus is given prior to CS-US pairings, a weaker CS-context association exists at the time of testing, which results in strong conditioned responding to the CS. This expression-failure view of the CS preexposure effect is supported by demonstrations of release from the CS preexposure effect, produced by posttraining extinction of the training context (Grahame et al., 1994; but see Hall \& Minor, 1984), context shifting between preexposure and training (Channel \& Hall, 1983), reminder treatments (Kasprow, Catterson, Schachtman, \& Miller, 1984), and delayed testing (Kraemer, Hoffman, \& Spear, 1988; Kraemer, Randall, \& Carbary, 1991; Kraemer \& Roberts, 1984).

Overshadowing refers to attenuated conditioned responding to a CS as a consequence of being paired with the US in the presence of another (usually more salient) stimulus. The comparator hypothesis offers an explanation of overshadowing, as well as of other cue competition effects (e.g., blocking) that arise from the presence of a better predictor of the US (owing to higher saliency or prior training history with the US) that is compounded with the target CS during training. Within a comparator framework, compound training results in a robust association between the target (overshadowed) CS and the comparator (overshadowing) stimulus. At test, the direct US representation activated by the target CS is weak, as compared with the indirect US representation mediated by the overshadowing (comparator) stimulus. This results in an attenuation of the conditioned response to the target CS that would otherwise be observed. According to the comparator hypothesis, any manipulation that weakens either the target CS-comparator stimulus association or the comparator stimulus-US association should increase the magnitude of conditioned responding that is observed to the target CS. Support for this prediction has been provided by studies in which, following excitatory training, the comparator stimulus-US association has been deflated (i.e., extinction of the comparator stimulus). Deflation of the comparator stimulus has been shown to reverse the effects of blocking (Blaisdell, Gunther, \& Miller, 1999), overshadowing (Kaufman \& Bolles, 1981; Matzel, Schachtman, \& Miller, 1985; 
Table 1

Design Summary: Experiment 1

\begin{tabular}{lccccccc}
\hline \multicolumn{1}{c}{ Group } & Phase $1_{1}$ & Phase 2 & Phase $3_{2}$ & Phase 4 & Test $_{3}$ & Expected \\
\hline Cl.NoPre & $\mathrm{Y}-$ & $+/ \mathrm{X}-$ & $\mathrm{C}+$ & $\mathrm{X}+$ & $\mathrm{X}$ & $\mathrm{cr}$ \\
OV.NoPre & $\mathrm{Y}-$ & $+/ \mathrm{AX}-$ & $\mathrm{C}+$ & $\mathrm{X}+$ & $\mathrm{X}$ & $\mathrm{CR}$ \\
Cl.Pre & $\mathrm{X}-$ & $+/ \mathrm{X}-$ & $\mathrm{C}+$ & $\mathrm{X}+$ & $\mathrm{X}$ & $\mathrm{cr}$ \\
OV.Pre & $\mathrm{X}-$ & $+/ \mathrm{AX}-$ & $\mathrm{C}+$ & $\mathrm{X}+$ & $\mathrm{X}$ & $\mathrm{cr}$ \\
Retard.Con & $\mathrm{Y}-$ & $+/ \mathrm{A}-$ & $\mathrm{C}+$ & $\mathrm{X}+$ & $\mathrm{X}$ & $\mathrm{CR}$ \\
GenDec & $\mathrm{Y}-$ & $+/ \mathrm{X}-$ & $\mathrm{C}+$ & $\mathrm{X}+$ & $\mathrm{AX}$ & $\mathrm{cr}$ \\
\hline
\end{tabular}

Note-Subscripts denote context. CI, conditioned inhibition; $O V$, overshadowing; Retard.Con, retardation control; GenDec, stimulus generalization decrement control; NoPre, no CS preexposure; Pre, CS preexposure. $\mathrm{X}$ and $\mathrm{Y}$ denote two stimuli (tone or white noise), counterbalanced within groups. A was a flashing light. $\mathrm{C}$ was a click train + and - denote the presence and absence of the US (footshock), respectively. / denotes explicitly unpaired. Transfer excitor training $(\mathrm{C}+)$ was given in order to equate stimuli exposure with that of Experiment 2. CR denotes expected strong conditioned responding; $c$ r denotes expected weak conditioned responding.

Matzel, Shuster, \& Miller, 1987), and the relative stimulus validity effect (Cole, Barnet, \& Miller, 1995).

Blaisdell et al. (1998) examined the interaction of CS preexposure treatment and overshadowing treatment on conditioned excitation, discovering that the two normally response-attenuating treatments counteracted each other, producing strong excitatory responding to the target CS The present series of experiments was designed to explore the nature of the interaction between stimulus preexposure and overshadowing within a conditioned inhibition preparation. Specifically, we attempted to replicate the interaction of preexposure and overshadowing, using an explicitly unpaired conditioned inhibition procedure (i.e., US/X - , with CS X being an initially neutral audiovisual stimulus and US a footshock). An explicitly unpaired conditioned inhibition procedure was chosen, rather than other standard procedures for producing conditioned inhibition (e.g., Pavlov, 1927) because, according to the comparator hypothesis, explicitly unpaired conditioned inhibition treatment establishes the context as the comparator stimulus for the target inhibitory CS X (see, e.g., Friedman, Blaisdell, Escobar, \& Miller, 1998). Thus, preexposure to $\mathrm{CS} X$ in the training context should strengthen the $\mathrm{X}$ comparator stimulus association. Unlike its effect on conditioned inhibition that is produced using the procedure of Pavlov (A-US/AX - , in which CS A is the training excitor), such preexposure has been shown to have no detrimental effect on the expression of explicitly unpaired conditioned inhibition to CS X (Friedman et al., 1998).

The present experiments used a $2 \times 2$ factorial design to manipulate CS preexposure and overshadowing of conditioned inhibition (see Table 1). In the first phase of training, the rats received preexposure to either CS $X$ or CS Y. This was followed by explicitly unpaired conditioned inhibition training in Phase 2, which was expected to result in behavior indicative of conditioned inhibition to CS X. During Phase 2, the rats received explicitly unpaired conditioned inhibition training with CS $\mathrm{X}$ alone (i.e., US/X-) or with an AX compound (with CS A

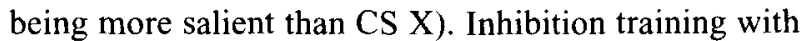
the AX compound was expected to result in CS A overshadowing CS $\mathrm{X}$ in becoming an effective inhibitor for subjects that had not received preexposure to CS $X$. That is, with only irrelevant treatment with CS Y in Phase 1, US/AX - training (in which the US and the AX compound presentations were interspersed with the US in an explicitly unpaired fashion) was expected to have the result that CS A, rather than the context, would become the comparator stimulus for CS X, thus disrupting the expression of conditioned inhibition to CS X. The preexposure treatment with CS X was intended to establish the context, rather than CS A, as the comparator stimulus for CS X (analogous to the preexposure-overshadowing condition of Blaisdell et al., 1998); thus, no overshadowing was anticipated in these subjects. Consequently, in this case, CS X was expected to pass both retardation (Experiment 1) and summation (Experiment 2) tests of conditioned inhibition, as a result of the preexposure treatment effectively counteracting the overshadowing treatment. In the General Discussion section, we elaborate on the presumed mechanism responsible for competition between CS A and the context for comparator status.

\section{EXPERIMENT 1 Retardation Test}

In Experiment 1, we used a retardation test to investigate whether pretraining exposure to a target CS (X) would prevent overshadowing of that $\mathrm{CS}$ by a stimulus of relatively high salience (A) that was trained in compound with CS X during explicitly unpaired inhibitory conditioning. CS preexposure treatment was used to establish the context, and not the putative overshadowing stimulus (A), as the comparator for CS X. In different groups, Phase 1 consisted of CS preexposure to the target inhibitory CS (X) or to an irrelevant (control) stimulus (Y). During Phase 2, the two conditions of Phase 1 were subdivided, and the subjects were assigned to either overshadowing or overshadowing-control conditions. Overshadowing groups received treatment designed to allow overshadowing of explicitly unpaired conditioned inhibition (i.e., US/AX - , with the US being a footshock and CS A being a more salient cue than CS X), whereas control subjects received elemental explicitly unpaired conditioned inhibition treatment with CS X (US/X-). A retardation control (Retard.Con) group received explicitly unpaired presentations of CS A and the US (i.e., US/A). This group was intended to measure unimpeded acquisition of the X-US association, against which retardation owing to conditioned inhibition in the other groups could be assessed. All the groups then received retardation test pairings of CS $\mathrm{X}$ and the US (i.e., $\mathrm{X}-\mathrm{US}$ ) and were subsequently tested for conditioned responding to CS X. On the basis of the comparator hypothesis, we expected that (1) explicitly unpaired inhibition training (Group CI.NoPre) would exhibit a strong retardation effect, owing to unimpeded inhibition, (2) CS preexposure treat- 
ment prior to explicitly unpaired inhibition training (Group CI.Pre) would have no deleterious effect (and perhaps a facilitatory effect) on the expression of conditioned inhibition, (3) overshadowing of conditioned inhibition treatment (Group OV.NoPre) would degrade conditioned inhibition to CS X, and (4) CS preexposure treatment followed by overshadowing treatment (Group OV.Pre) would provide protection against overshadowing of conditioned inhibition, all with respect to a baseline for assessing conditioned inhibition (Group Retard.Con). An additional control group (GenDec) was included in order to determine the extent to which the overshadowing observed in our overshadowing preparation arose from stimulus generalization decrement as a result of training with the $A X$ compound and testing with $\mathrm{CS} X$ alone. According to contemporary models that explain overshadowing in terms of generalization decrement (e.g., Pearce, 1987), the decrement in responding to an element after training that element in a compound (i.e., overshadowing treatment) should be equivalent to the decrement in responding to a compound after training one element of that compound (e.g., Group GenDec of Experiment 1). Therefore, if overshadowing in our preparation was due to generalization decrement, we would expect little conditioned responding in Group GenDec. However, if overshadowing in our preparation was due to processes that treat the stimuli as elements (e.g., the comparator hypothesis), Group GenDec should demonstrate strong conditioned responding. Notably, the elements constituting our compounded cues were intentionally selected to minimize the likelihood of configuring; that is, they were of different modalities, or if of the same modality, they emanated from different locations within the apparatus.

\section{Method}

\section{Subjects}

Thirty-six male (281-424 g) and 36 female (220-282 g) naive, Sprague-Dawley descended rats, bred in our colony from Holtzman stock, served as subjects. The rats were randomly assigned to one of six groups $(n s=12)$, counterbalanced for sex. The subjects were individually housed in wire-mesh cages in a vivarium maintained on a 16:8-h light:dark cycle. Experimental manipulations occurred approximately midway through the light portion of the cycle. A progressive water deprivation schedule was imposed over the week prior to the beginning of the experiment until water availability was limited to $10 \mathrm{~min}$ per day. All the rats were handled for $30 \mathrm{sec}$ three times per week, from weaning until the initiation of the study.

\section{Apparatus}

Twelve identical chambers, each measuring $30 \times 25 \times 32 \mathrm{~cm}(1 \times$ $w \times h$ ), were used. The walls of each chamber were made of Plexiglas, and the floor was constructed of 0.5 -cm-diameter rods, spaced $2 \mathrm{~cm}$ center to center and connected by NE-2 neon bulbs that allowed a $0.7-\mathrm{mA}$ constant-present footshock to be delivered by means of a high voltage ac circuit in series with a $1.0-\mathrm{M} \Omega$ resistor. Each chamber was housed in an environmental isolation chest that was dimly illuminated by a houselight (1820 incandescent bulb) mounted on the ceiling of the experimental chamber. Each chamber was equipped with a water-filled lick tube (opening $=0.3 \mathrm{~cm}$ in diameter) that extended about $1 \mathrm{~cm}$ from the rear of a cylindrical niche, $4.5 \mathrm{~cm}$ in diameter, that was left-right centered on one wall, with its axis perpendicular to the wall and positioned $4 \mathrm{~cm}$ above the grid floor. An infrared photobeam was projected horizontally across the niche, $1 \mathrm{~cm}$ in front of the lick tube. In order to drink from the tube, the subjects had to insert their heads into the niche, thereby breaking the horizontal infrared photobeam. Thus, the amount of time the photobeam was disrupted could be monitored; this served as our dependent measure. A $45-\Omega$ speaker mounted on the interior back side of each environmental chest could deliver a high-frequency complex tone (T; 3000 and $3200 \mathrm{~Hz}$ ) $6 \mathrm{~dB}$ (C-scale) SPL above background. A second $45-\Omega$ speaker mounted on the ceiling of the experimental chamber was used to deliver a click (C) stimulus $(6 / \mathrm{sec}) 6 \mathrm{~dB}$ (C-scale) above background. A third $45-\Omega$ speaker, mounted on the sidewall of the chamber, was used to deliver a white noise (N) stimulus $6 \mathrm{~dB}$ (C-scale) above background. A 75$\mathrm{W}$ (nominal at $120 \mathrm{~V}$ ac but driven at $100 \mathrm{~V}$ ac) incandescent bulb mounted on the back wall of each environmental chest was able to deliver a flashing $(0.25 \mathrm{sec}$ on $/ 0.25 \mathrm{sec}$ off) light (L) CS. The tone and white noise, counterbalanced within groups, served as the target stimuli $\mathrm{X}$ and $\mathrm{Y}$. The flashing light always served as the overshadowing stimulus (A). The click train always served as Stimulus C, which, in Experiment 2, served as a transfer excitor. A 0.7-mA, $0.5-\mathrm{sec}$ footshock could be delivered through the chamber floors. All CSs were $10 \mathrm{sec}$ in duration, except at test. Ventilation fans in each enclosure provided a constant 76-dB (C-scale) background noise.

Three contexts were used: Context 1 for CS preexposure, conditioned inhibition, and retardation treatments; Context 2 for transfer excitor training; and Context 3 for testing. Context 1 was the described apparatus with the houselight turned on. Context 2 was created by using a different chamber than Context 1 for each subject and by turning off the houselight. Context 3 was created by using a different chamber than either Context 1 or Context 2 for each subject, turning on the houselight, placing Plexiglas floor plates over the grid floors, and providing an odor cue (two drops of $98 \%$ methyl salicylate on a wooden block placed inside the environmental enclosure). The second context was used for transfer excitor training, with the intent of minimizing any additional training context-US experience that might influence the expression of conditioned inhibition to CS X. The third context was used for testing, in order to avoid testing the subjects in the conditioned inhibition training context, because it was likely that the training context would be highly excitatory, or in the transfer excitor context, because research has shown that behavior indicative of inhibition does not readily transfer to a context in which signaled USs have been presented (i.e., the renewal effect; see, e.g., Bouton \& Bolles, 1979; Bouton \& King, 1983).

\section{Procedure}

The critical aspects of the experimental procedure are summarized in Table 1. All the sessions were $60 \mathrm{~min}$ in duration, except where otherwise noted.

Acclimation. On Day 1, each subject received four nonreinforced presentations of stimuli light $(\mathrm{L})$, tone $(\mathrm{T})$, and noise $(\mathrm{N})$, with a mean intertrial interval of $4 \mathrm{~min}$ (range $=2-8 \mathrm{~min}$, in the following order: $L, T, N, T, N, L, N, L, T, N, T, L$ ), in Context 1 . These brief preexposures to each cue were intended to reduce unconditioned responding to any cue during testing. Without this preexposure procedure, Group GenDec would have experienced CS A for the first time during testing, thereby making unconditioned suppression of drinking likely. On Day 2, the subjects received, in Context 2, similar treatment as that on Day 1, except that there were no nominal stimulus presentations. Lick tubes were absent on both days. On Day 3, all subjects were acclimated to Context 3 as on Day 1, except that lick tubes were in place. All subjects again received four nonreinforced presentations of Stimuli L, T, and N, as on Day 1 .

Phase 1 (CS preexposure). Prior to the initiation of Phase 1, the lick tube was removed from each chamber. On Days 4-11, subjects 
received 60 daily presentations of the $X$ stimulus (Groups CI.Pre and OV.Pre) or the Y stimulus (Groups CI.NoPre, OV.NoPre, Retard.Con, and GenDec) in Context 1 , with a mean intertrial interval of $60 \mathrm{sec}$ (range $=30-120 \mathrm{sec}$ ), during these daily 60 -min sessions, for a total of 480 presentations of CS X or CS Y.

Phase 2 (conditioned inhibition). On Days $12-17$, subjects in Groups OV.NoPre and OV.Pre received 44 unsignaled shock presentations, interspersed with 4 nonreinforced AX compound presentations per daily 60-min session in Context 1 (for a total of 24 $\mathrm{AX}$ - presentations), with a mean intertrial interval of $60 \mathrm{sec}$ (range $=30-90 \mathrm{sec}$ ). All $4 \mathrm{AX}$ compound presentations were preceded and followed by at least $60 \mathrm{sec}$ of shock-free time. All other subjects similarly received 44 unsignaled shock presentations, interspersed with 4 nonreinforced CS X (Groups CI.NoPre, CI.Pre, and GenDec) or CS A (Group Retard.Con) presentations per daily session, for a total of $24 \mathrm{CS} \mathrm{X}$ - or CS A - presentations.

Phase 3 (transfer excitor training). On Days 18 and 19, all subjects received two reinforced presentations of CS C (the clicks), with a mean intertrial interval of $20 \mathrm{~min}$ per daily 40 -min session. Although no transfer test was conducted in this experiment, transfer excitor training was administered in order to equate treatments with those in Experiment 2.

Phase 4 (retardation [X $\rightarrow$ US] training). On Day 20, all subjects received three $X \rightarrow$ US pairings (with US onset immediately following CS termination), with a mean intertrial interval of $15 \mathrm{~min}$ in Context 1 .

Reacclimation. On Days 21-24, the lick tubes were reinserted in Context 3, and subjects were allowed to drink during each session. This treatment served to restabilize baseline levels of drinking. These sessions did not include any nominal stimulus presentations.

Testing. On Day 25, subjects in Groups CI.NoPre, OV.NoPre, CI.Pre, OV.Pre, and Retard.Con were tested in Context 3 for conditioned lick suppression to CS X by presenting CS X immediately on completion of 5 cumulative sec of licking (as measured by the total amount of time the infrared photobeam was disrupted). Thus, all subjects were drinking at the time of CS onset. Subjects in Group GenDec were similarly tested for conditioned lick suppression to the AX compound. The time to complete this initial 5 cumulative sec of licking and the time to complete an additional 5 cumulative sec of licking after the onset of the test $\mathrm{CS}$ (or AX compound for Group GenDec) were recorded. Test sessions were $16 \mathrm{~min}$ in duration, with a ceiling score of $15 \mathrm{~min}$ being imposed on the time to complete 5 cumulative sec of drinking in the presence of the test CS.

Suppression data were transformed to $\log$ (base 10) scores, to facilitate the use of parametric statistics. An alpha level of $p=.05$ was adopted for all tests of statistical significance. In addition, following the convention of our laboratory, subjects that required more than $60 \mathrm{sec}$ to complete their first 5 cumulative sec of licking (i.e., prior to CS onset) were eliminated from all analyses, because their behavior suggested unusually high fear of the test context. Two subjects met this criterion ( 1 from Group CI.NoPre and 1 from Group CI.Pre); thus, their data were eliminated from all the analyses.

\section{Results and Discussion}

The central observations from Experiment 1 were that (1) explicitly unpaired conditioned inhibition treatment (Group CI.NoPre) produced conditioned inhibition, as assessed by a retardation test; (2) CS A overshadowed CS X in acquiring conditioned inhibition (Group OV.NoPre); and (3) preexposure to CS X in the training context prior to overshadowing training (Group OV.Pre) prevented CS A from overshadowing CS X (see Figure 2). Furthermore, preexposure to CS X (Group CI.Pre) had no demon- strable effect on the expression of conditioned inhibition to CS X. The following analyses support these conclusions.

A one-way analysis of variance (ANOVA) conducted on pre-CS times to complete 5 cumulative sec of licking found no significant group differences in baseline drinking behavior in this experiment $(F<1)$. This suggests a similar behavioral baseline across groups. A one-way analysis of covariance (ANCOVA) conducted on suppression scores during the test CS or compound, with sex (male or female), stimulus X (tone or noise), and log pre$\mathrm{CS}$ scores as covariates, revealed a treatment effect $\left[F(5,61)=20.29, M S_{\mathrm{e}}=0.058, p<.001\right]$. (Analysis of Experiment 1 without these covariates yielded the same significant differences. The covariates were used here for consistency with Experiment 2, in which an ANCOVA was necessary in order to factor out some of the unusually high within-group variance that interfered with our detecting significant group differences.) A $2 \times 2$ ANCOVA, with Phase 1 treatment (stimulus: $\mathrm{X}$ or $\mathrm{Y}$ ) and Phase 2 treatment (stimulus: $\mathrm{AX}$ or $\mathrm{X}$ ) as factors, was conducted on suppression scores from the first four groups (Groups CI.NoPre, OV.NoPre, CI.Pre, and OV.Pre). This revealed a main effect of Phase 1 treatment $\left[F(1,39)=24.14, M S_{\mathrm{e}}=\right.$ $0.058, p<.001$ ], a main effect of Phase 2 treatment $\left[F(1,39)=17.54, M S_{\mathrm{e}}=0.058, p<.001\right]$, and an interaction $\left[F(1,39)=19.67, M S_{\mathrm{e}}=0.058, p<.001\right]$.

Planned comparisons were conducted on the suppression to CS X scores, using the overall error term from the one-way ANCOVA. Subjects in Group CI.NoPre suppressed less than did the subjects in Group Retard.Con, demonstrating conditioned inhibition (as seen in a retardation test) with an explicitly unpaired preparation $\left[F(1,61)=29.43, M S_{\mathrm{e}}=0.76, p<.001\right]$. Moreover, the presence of CS A during conditioned inhibition training (Group OV.NoPre) led to greater suppression to CS X than did the absence of CS A (Group CI.NoPre), thereby indicating overshadowing of CS X by CS A $[F(1,61)=$ $\left.28.19, M S_{\mathrm{e}}=0.076, p<.001\right]$. However, preexposure to CS X prior to overshadowing training (Group OV.Pre) attenuated overshadowing, as compared with cases in which no preexposure to $\mathrm{CS} X$ was given [Group OV.NoPre; $\left.F(1,61)=33.48, M S_{\mathrm{e}}=0.076, p<.001\right]$. Group GenDec suppressed less than did Group OV.NoPre $[F(1,61)=$ $\left.8.73, M S_{\mathrm{e}}=0.076, p<.01\right]$, demonstrating that the weak suppression to CS X observed in the overshadowing group was not due to stimulus generalization decrement. Thus, preexposure to a target CS appears to have protected it from overshadowing during subsequent explicitly unpaired conditioned inhibition treatment. One might expect, on the basis of the comparator hypothesis, that CS preexposure would enhance the amount of conditioned inhibition observed with the explicitly unpaired procedure. That is, the comparator hypothesis explains conditioned inhibition produced with the explicitly unpaired procedure as being the result of a strong association between CS X and the excitatory context (i.e., link 2 of Figure 1). Thus, CS preexposure should, if anything, strengthen the $\mathrm{X}$-context association, thereby enhancing the magnitude of condi- 


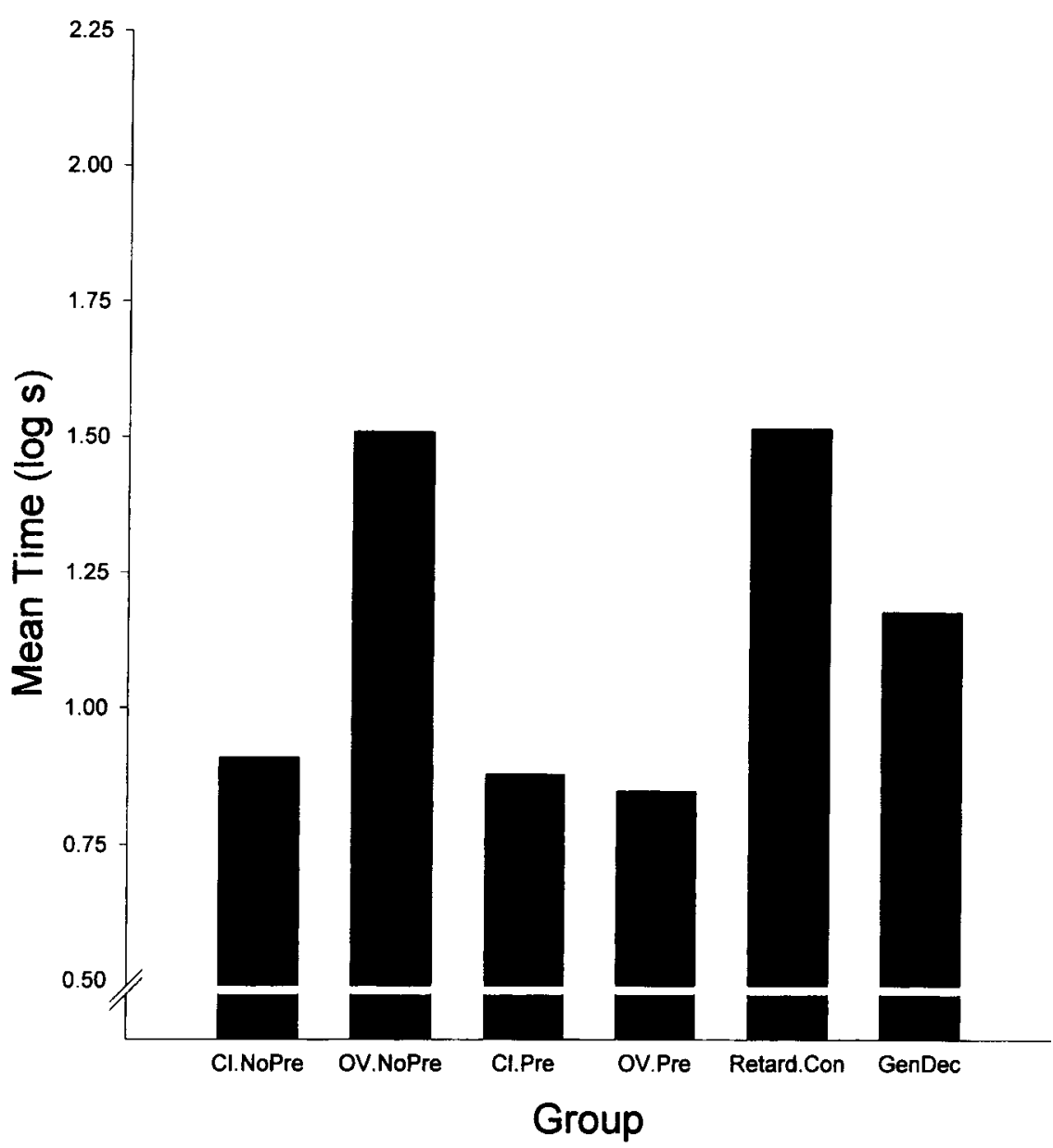

Figure 2. Experiment 1: ANCOVA-adjusted mean times $(\log$ sec) to complete 5 cumulative sec of licking in the presence of the target conditioned stimulus. All the groups were tested on X, except Group GenDec, which was tested on AX.

tioned inhibition observed. However, as Friedman et al. (1998) have noted, such an enhancement of conditioned inhibition would be observed only if the absolute value of the CS X-context association acquired during conditioned inhibition training had not reached asymptote. Both Friedman et al. and the present experiment gave extensive conditioned inhibition training, making it highly likely that the $\mathrm{X}$-context association in the conditioned inhibition alone group (Group CI.NoPre) resulted in the acquisition of an asymptotic $\mathrm{X}$-context association. Therefore, there is little reason to expect any additional effect of preexposure to the $\mathrm{CS}$ alone on behavior indicative of conditioned inhibition. Thus, we did not perform any planned comparisons involving the CS preexposure group (Group CI.Pre).

\section{EXPERIMENT 2 Summation Test}

Experiment 1 demonstrated the preexposure-overshadowing effect (Blaisdell et al., 1998) in conditioned inhibition, as assessed by a retardation test. In Group OV.NoPre, CS A overshadowed CS X, suggesting that CS A, rather than the context, was the comparator stimulus for CS X. In Group OV.Pre, preexposing CS X prior to overshadowing appeared to establish the context as the comparator stimulus for CS X, thereby preventing CS A from overshadowing CS X. However, an alternative account of the weak conditioned responding to CS X observed in Group OV.Pre of Experiment 1 (i.e., retardation) is that preexposure to CS X may have resulted in latent inhibition. It is generally accepted that latent inhibitors pass a retardation test for behavioral control but fail to pass a summation test (Cole, Barnet, \& Miller, 1997; Hearst, 1972; Reiss \& Wagner, 1972; Rescorla, 1971; but see Papini \& Bitterman, 1993). Therefore, in Experiment 2, we used a summation test for conditioned inhibition in order to further examine the conjoint effects of CS preexposure and overshadowing treatments on the development of explicitly unpaired conditioned inhibition. The same predictions as those outlined for Experiment 1 apply to Experiment 2 . 
Table 2

Design Summary: Experiment 2

\begin{tabular}{lllclc}
\hline \multicolumn{1}{c}{ Group } & Phase $1_{1}$ & Phase $2_{1}$ & Phase $3_{2}$ & Test $_{3}$ & Expected \\
\hline CI.NoPre & $\mathrm{Y}-$ & $+/ \mathrm{X}-$ & $\mathrm{C}+$ & $\mathrm{CX}$ & $\mathrm{cr}$ \\
OV.NoPre & $\mathrm{Y}-$ & $+/ \mathrm{AX}-$ & $\mathrm{C}+$ & $\mathrm{CX}$ & $\mathrm{CR}$ \\
CI.Pre & $\mathrm{X}-$ & $+/ \mathrm{X}-$ & $\mathrm{C}+$ & $\mathrm{CX}$ & $\mathrm{cr}$ \\
OV.Pre & $\mathrm{X}-$ & $+/ \mathrm{AX}-$ & $\mathrm{C}+$ & $\mathrm{CX}$ & $\mathrm{cr}$ \\
Sum.Con & $\mathrm{Y}-$ & $+/ \mathrm{X}-$ & $\mathrm{C}+$ & $\mathrm{C}$ & $\mathrm{CR}$ \\
GenDec & $\mathrm{Y}-$ & $+/ \mathrm{X}-$ & $\mathrm{C}+$ & $\mathrm{CAX}$ & $\mathrm{cr}$ \\
\hline
\end{tabular}

Note-Subscripts denote context. CI, conditioned inhibition; OV, overshadowing; Sum.Con, summation control; GenDec, stimulus generalization decrement control; NoPre, no CS preexposure; Pre, CS preexposure. $\mathrm{X}$ and $\mathrm{Y}$ denote two stimuli (tone or white noise), counterbalanced within groups. A was a flashing light. $C$ was a click train. + and - denote the presence and absence of the US (footshock), respectively. / denotes explicitly unpaired. CR denotes expected strong conditioned responding; cr denotes expected weak conditioned responding.

\section{Method}

\section{Subjects and Apparatus}

Thirty-six male (350-480 g) and 36 female (210-335 g) naive, Sprague-Dawley descended rats, bred in our colony from Holtzman stock, served as subjects. The animals were randomly assigned to one of six groups ( $n \mathrm{~s}=12$ ), counterbalanced within group for sex. The animals were housed and maintained as were those in Experiment 1 . The apparatus and stimuli were identical to those used in Experiment 1.

\section{Procedure}

Specific group names are explained, along with a summary of the critical aspects of the training procedure, in Table 2 . All sessions were $60 \mathrm{~min}$ in duration, except where otherwise noted.

Acclimation. On Days 1-3, all subjects were acclimated to Contexts $1-3$ in the same way as that in Experiment 1 .

Phase 1 (CS preexposure). On Days 4-11, all subjects in Groups CI.NoPre, OV.NoPre, CI.Pre, OV.Pre, and GenDec received training in Context 1 in the same way as that in Experiment 1. Group Sum.Con received training identical to that for Group Retard.Con of Experiment 1.

Phase 2 (conditioned inhibition). On Days 12-17, all groups except Sum.Con received treatment in Context 1 identical to that received by the correspondingly named groups in Experiment 1. Group Sum.Con received treatment identical to that for Group $\mathbf{R e}$ tard.Con, except that the subjects received nonreinforced presentations to CS X, rather than to CS A.

Phase 3 (transfer excitor training). On Days 18 and 19, all subjects received transfer excitor training in Context 2 in the same way as that in Experiment 1.

Reacclimation. On Days 20-23, all subjects were reacclimated to Context 3 in the same way as that in Experiment 1.

Testing. On Day 24, subjects in Groups CI.NoPre, OV.NoPre, CI.Pre, and OV.Pre were tested in Context 3 for conditioned lick suppression to the CX compound. Testing was conducted in Context 3 in the same manner as that in Experiment 1. Subjects in Group Sum.Con were tested in a similar manner on the $\mathrm{C}$ element alone, whereas subjects in Group GenDec were tested on the CAX compound. One rat from Group OV.Pre failed to complete its first 5 cumulative sec of licking in $60 \mathrm{sec}$; thus, its data were eliminated from all the analyses.

\section{Results and Discussion}

Experiment 2 successfully replicated the critical outcomes of Experiment 1. The central observations from Experiment 2 were that (1) explicitly unpaired conditioned inhibition treatment (Group CI.NoPre) produced conditioned inhibition, as assessed by a summation test; (2) CS A overshadowed CS X in acquiring conditioned inhibition (Group OV.NoPre); and (3) preexposure to CS X in the training context prior to overshadowing training (Group OV.Pre) prevented CS A from overshadowing CS X (Figure 3). Furthermore, preexposure to CS X (Group CI.Pre) had no detrimental effect on the expression of conditioned inhibition to CS X. The following analyses support these conclusions.

A one-way ANOVA, conducted on pre-CS times to complete 5 cumulative sec of licking, found no significant group differences in baseline drinking behavior in this experiment $(F<1)$. A one-way ANCOVA, conducted on suppression scores during the test CS or compound, with sex (male or female), stimulus X (tone or noise), and log pre-CS scores as covariates, revealed a treatment effect $\left[F(5,62)=6.08, M S_{\mathrm{e}}=0.197, p<.001\right]$. A $2 \times 2 \mathrm{AN}-$ COVA, with Phase 1 treatment (stimulus: $X$ or $Y$ ) and Phase 2 treatment (stimulus: $\mathrm{AX}$ or $\mathrm{X}$ ) as factors, was conducted on suppression scores from the first four groups (Groups CI.NoPre, OV.NoPre, CI.Pre, and OV.Pre). This revealed a main effect of Phase 1 treatment $[F(1,42)=$ $\left.11.32, M S_{\mathrm{e}}=0.231, p<.01\right]$ and an interaction $[F(1,42)=$ $\left.6.96, M S_{\mathrm{e}}=0.231, p<.05\right]$. The main effect of Phase 2 treatment was not significant $(F<1)$.

Planned comparisons were conducted on the suppression to CS X scores, using the overall error term from the one-way ANCOVA. Subjects in Group CI.NoPre suppressed less to the CX compound than subjects in Group Sum.Con suppressed to CS C, thereby demonstrating conditioned inhibition with an explicitly unpaired preparation $\left[F(1,62)=7.1, M S_{\mathrm{e}}=0.197, p<.01\right]$. Moreover, the presence of CS A during conditioned inhibition training (Group OV.NoPre) led to greater suppression to the CX compound than did the absence of CS A during inhibition training (Group CI.NoPre), thereby indicating overshadowing of CS X by CS A $\left[F(1,62)=8.06, M S_{\mathrm{e}}=\right.$ $0.197, p<.01]$. However, preexposure to CS X prior to overshadowing training (Group OV.Pre) attenuated overshadowing of CS X by CS A, as compared with cases in which no preexposure to $\mathrm{CS} X$ was given [Group OV.NoPre; $\left.F(1,62)=18.31, M S_{\mathrm{e}}=0.197, p<.001\right]$. Furthermore, the potential of CS X in Group OV.Pre to lower suppression to CS C, relative to Group OV.NoPre, suggests that, in Experiment 1, the behavior of Group OV.Pre was not induced by latent inhibition, as opposed to conditioned inhibition. Suppression to the CX compound by Group OV.NoPre was marginally greater than that to the CAX compound by Group GenDec $\left[F(1,62)=3.72, M S_{\mathrm{e}}=\right.$ $0.197, p=.058$ ], demonstrating that overshadowing in our preparation was not produced by stimulus generalization decrement. The failure to demonstrate significantly weaker suppression in Group GenDec than in Group OV.NoPre was probably due to disinhibition (Pavlov, 1927), induced by the presence of the relatively novel A stimulus (a bright flashing light) along with the highly excitatory $\mathrm{C}$ stimulus. 


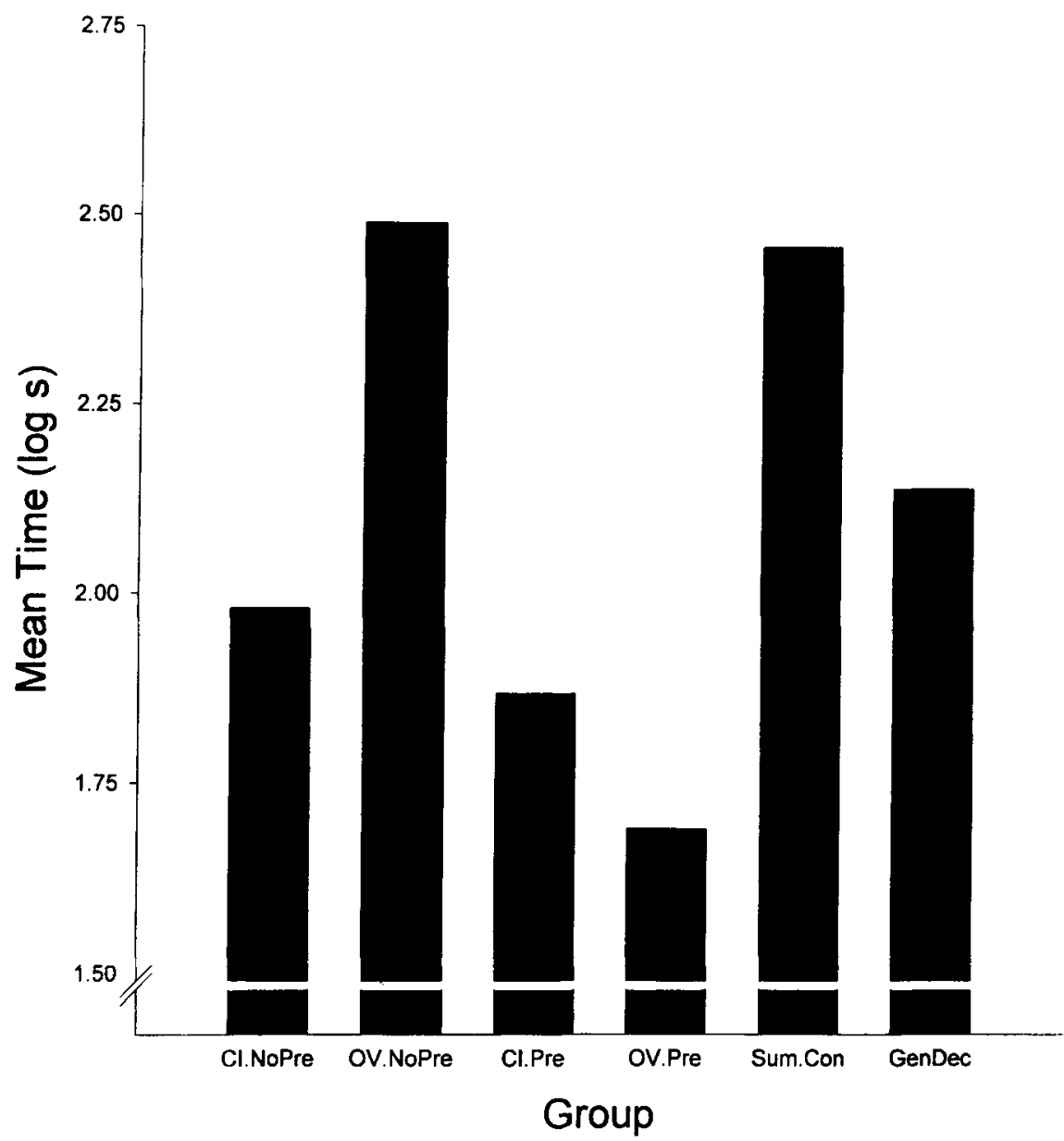

Figure 3. Experiment 2: ANCOVA-adjusted mean times (log sec) to complete 5 cumulative sec of licking in the presence of the target conditioned stimulus. All the groups were tested on CX, except for Group Sum.Con, which was tested on C, and Group GenDec, which was tested on CAX.

An alternative account of conditioned inhibition in a summation test is that testing on the CX compound in Group CI.NoPre resulted in generalization decrement in excitatory responding to CS C, relative to Group Sum.Con. However, if such an interpretation were viable, the same or more generalization decrement should have been observed in Group OV.NoPre, which was also tested on the $\mathrm{CX}$ compound. However, strong excitatory responding was seen in this latter group, discounting a generalization decrement interpretation of our demonstration of conditioned inhibition via a summation test.

A similar argument in terms of generalization decrement could be made for the strong responding by Group OV.NoPre in both Experiment 1 and Experiment 2. That is, if subjects automatically configure stimuli presented in compound, the $\mathrm{AX}$ compound presented during conditioned inhibition training of Phase 2 would be perceived as being highly different than CS X during Phase 4 and the test phase of Experiment 1 and during the test phase of Experiment 2. Such configuring of stimuli would make
CS X effectively novel during Phase 4 of Experiment 1 and during testing of Experiment 2, and a novel stimulus would not be expected to pass retardation or summation tests of conditioned inhibition. Although this is a viable account of responding by Group OV.NoPre in Experiment 1, in Experiment 2, the strong conditioned inhibition observed in Group GenDec weakens a generalization decrement interpretation of the overshadowing of conditioned inhibition demonstrated in Group OV.NoPre.

\section{GENERAL DISCUSSION}

The present experiments demonstrated overshadowing of explicitly unpaired conditioned inhibition. That is, the presence of a more salient stimulus (A) in compound with a less salient stimulus $(\mathrm{X})$ during explicitly unpaired conditioned inhibition training prevented (i.e., overshadowed) CS X from passing retardation (Experiment 1) and summation (Experiment 2) tests for conditioned inhibition. However, pretraining exposure to CS X prior to ex- 
plicitly unpaired conditioned inhibition training prevented CS A from overshadowing CS X (i.e., the preexposureovershadowing effect). This finding extends to inhibitory behavior the finding of the interaction between CS preexposure treatment and overshadowing treatment observed with an excitatory preparation (Blaisdell et al., 1998). It should also be noted that the present experiments provide the first known demonstration of overshadowing of conditioned inhibition (see Suiter \& LoLordo, 1971, for a demonstration of blocking of conditioned inhibition).

Although neither conventional associative models of learning that emphasize acquisition (e.g., Pearce \& Hall, 1980; Rescorla \& Wagner, 1972) nor the comparator hypothesis in its original form (e.g., Miller \& Matzel, 1988; Miller \& Schachtman, 1985) provide an adequate account of the preexposure-overshadowing effect, we recently presented an extension of the comparator hypothesis (Blaisdell et al., 1998; Friedman et al., 1998) that, among other things, predicts the combined effects of CS preexposure and overshadowing. The extended comparator hypothesis makes the unique and counterintuitive prediction that these two treatments will counteract each other.

In situations involving multiple nontarget stimuli, such as many cue competition paradigms in which nontarget punctate cues, along with the training context, are present during training of the target $\mathrm{CS}$, there is the potential for competition between these stimuli in serving as the comparator stimulus for the target CS. Preliminary research has demonstrated that such comparator competition indeed occurs and has uncovered some of the important variables that influence comparator status (e.g., Barnet, Grahame, \& Miller, 1993b; Blaisdel et al., 1998; Denniston, Savastano, Blaisdell, \& Miller, 1999; Friedman et al., 1998; Grahame, Barnet, \& Miller, 1992; Miller, Esposito, \& Grahame, 1992). These variables include relative saliency (Miller et al., 1992), relative contiguity with the target CS (Barnet, Grahame, \& Miller, 1993a), and relative time in training history (Barnet et al., 1993b; Blaisdell et al., 1998; Friedman et al., 1998; Grahame et al., 1992).

To account for the way these variables influence successful establishment of comparator stimuli for a target $\mathrm{CS}$, the extended comparator hypothesis postulates that the degree to which a nontarget stimulus comes to serve as the comparator stimulus for the target CS depends on the effective strength of the within-compound association between the CS and the comparator stimulus at the time of testing. The strength of within-compound associations between the target CS and candidate comparator stimuli presumably depend on (1) the saliency of the nontarget stimulus, (2) the number of pairings between the target CS and the nontarget stimulus, (3) the relative recency in training with which the nontarget stimulus was paired with the target CS, and (4) the spatial and temporal proximity of the nontarget stimulus with the target $C S$ (i.e., contiguity, with a stronger within-compound association forming with increasing contiguity). Competition between multiple nontarget stimuli to serve as the com- parator stimulus for the target CS depends on the relative strength of the within-compound association between each candidate comparator stimulus and the target CS. Thus, the greater the difference in magnitude of the within-compound association with the target CS possessed by two nontarget stimuli, the greater the role of the nontarget stimulus with the stronger within-compound association in serving as the comparator stimulus (see, e.g., Miller et al., 1992, in which only extinction of the more salient of two candidate comparator stimuli [punctate stimulus or context, for which the relative salience was varied] produced a change in responding to the target $C S$ ). In situations in which two nontarget stimuli have withincompound associations of similar strengths, both will act (approximately) equally as comparator stimuli. This has been shown by Grahame et al. (1992), in which extinction of both of two local context cues (of equal salience) was required to influence responding to the target CS (see, also, Miller et al., 1992).

The difference in relative strengths of the withincompound association each candidate comparator stimulus has with the target CS has been shown to depend on the number of pairings each nontarget CS has had with the target CS. For example, Blaisdell et al. (1998) and Friedman et al. (1998) gave many context-target CS pairings prior to overshadowing and Pavlovian conditioned inhibition training, respectively. Such extensive contextCS pairings should establish a strong within-compound association between the context and the target CS. Subsequent presentations of the target CS with the US (Blaisdell et al., 1998) or without the US (Friedman et al., 1998) in the presence of another punctate stimulus (A) also established a within-compound association between CS X and CS A. However, because there were far fewer X-A pairings than $\mathrm{X}$-context pairings, the within-compound association between CS A and CS X should have been relatively weaker than that between the context and CS X. Therefore, the context, and not CS A, should have served as the comparator stimulus for CS X. Blaisdell et al. (1998) demonstrated this by showing that associative inflation of the context, but not of CS A, had an effect on excitatory responding to $\mathrm{CS} X$.

In the experiments reported here, the extended comparator hypothesis predicts that CS preexposure treatment will attenuate overshadowing in a manner similar to that described in the preceding paragraph. Specifically, because there were many CS preexposure trials (i.e., many $\mathrm{X}$-context pairings) and relatively few $\mathrm{AX}$ trials (i.e., few A-X pairings) for Group OV.Pre, CS X should have formed a stronger within-compound association with the context than with the overshadowing stimulus (CS A). Consequently, the context (rather than the overshadowing stimulus [CS A]) should have acted as the comparator stimulus for the preexposed target CS (X). The context should have been highly excitatory, as a result of the unsignaled USs administered during explicitly unpaired conditioned inhibition training. At test, the weak (or nonexistent) US representation activated directly by CS X 
was presumably compared with the strong US representation activated indirectly by the excitatory context, thereby resulting in behavior indicative of conditioned inhibition in response to CS X. Thus, the comparator hypothesis predicts that, when CS preexposure precedes overshadowing treatment embedded within an explicitly unpaired conditioned inhibition procedure, the preexposure treatment should counteract the effects of the overshadowing treatment.

In the absence of preexposure to the target CS, the comparator hypothesis predicts that overshadowing of a conditioned inhibitor will occur when the target $\mathrm{CS}$ undergoes explicitly unpaired conditioned inhibition treatment in compound with another, more salient potential inhibitor. The nontarget element of the compound (CS A), being highly salient, becomes the comparator stimulus of the target inhibitor (CS X), thereby preventing the (excitatory) training context from acting as the comparator stimulus for CS X. At test, the US representation that is presumed to be directly activated by CS X through the X-US association is compared with the US representation that is indirectly activated by CS $X$ through the conjoint actions of the $\mathrm{X}-\mathrm{A}$ and $\mathrm{A}-\mathrm{US}$ associations. Behavior indicative of CS X being a conditioned inhibitor is assumed to be inversely related to the strength of the X-US association and directly related to the relative strength of the product of the X-A and A-US associations. After explicitly unpaired conditioned inhibition training of the $A X$ compound (US/AX), both the $\mathrm{X}$-US and the A-US associations should be negligible, despite the strong $X-A$ association. Thus, no conditioned inhibition to CS X should be observed.

According to most contemporary models of learning that emphasize acquisition, CS preexposure should either have little effect on subsequent learning about the CS (e.g., Rescorla \& Wagner, 1972) or should facilitate overshadowing of the less salient target CS by the more salient stimulus (e.g., Pearce \& Hall, 1980). The RescorlaWagner model predicts overshadowing of conditioned inhibition, albeit through a different mechanism than that suggested by the comparator hypothesis. In the Rescorla-Wagner model, CS A competes with CS X in acquiring negative associative strength, thereby resulting in CS $X$ acquiring little (negative) associative strength. Thus, the Rescorla-Wagner model treats overshadowing of conditioned inhibition as being completely symmetrical to overshadowing of conditioned excitation-only with negative, rather than positive, associative strengths. The Rescorla-Wagner model and the comparator hypothesis diverge, however, in their predictions regarding the effects of CS preexposure treatment on overshadowing of conditioned inhibition. The Rescorla-Wagner model predicts no effect of preexposing CS $\mathrm{X}$ on subsequent overshadowing of CS X, because changes in the associative strength of a stimulus are assumed not to occur until a US is presented or an expected US is omitted. During preexposure to CS X, no US is presented, and no expected US is omitted; thus, no change in associative strength of
CS X is anticipated. This prediction is in accord with the noted failure of the Rescorla-Wagner model to explain latent inhibition (Miller, Barnet, \& Grahame, 1995). This failure is also seen in the revised Rescorla-Wagner model (Van Hamme \& Wasserman, 1994), which was devised to account for some of the failings of the original RescorlaWagner model and in which the associability of absent cues (i.e., cues not present on a particular trial) is nonzero. This modification, which assigns to absent cues a negative associability, allows the revised model to account for the effects of posttraining changes in a competing stimulus' associative strength through a process of retrospective revaluation of the absent cues' associative strength.

Associative models that can account for both the CS preexposure effect and the overshadowing deficit predict that the two treatments should summate to produce a larger deficit in responding to the CS than would either treatment alone (e.g., Pearce \& Hall, 1980). More generally, attentional interpretations of cue competition effects (e.g., Lubow et al., 1976; Lubow et al., 1981; Mackintosh, 1975; Pearce \& Hall, 1980) fail to explain how preexposure and overshadowing treatments would interact so that they would counteract the deficit anticipated to result from each individual treatment. That is, if CS preexposure and overshadowing treatments each resulted in a decreased attention to the CS, combining such treatments should only enhance such losses of attention paid to the CS, thereby resulting in a summation of the deficits in responding produced by each treatment.

Another acquisition-focused account of the CS preexposure effect is provided by Wagner's (1981) SOP model of Pavlovian conditioning. This model assumes that retarded acquisition of conditioned responding to a CS that has been preexposed is attributable to the context activating the $A 2$ (i.e., secondary activity) state of the CS representation at the beginning of the conditioning session(s), thereby limiting the amount of Al (i.e., primary activity) state that the CS itself can activate. Because acquisition of conditioned responding to the $C S$ requires that the CS representation be in the Al state during presentation of the US, acquisition of conditioned responding to a preexposed CS should be retarded. This same mechanism can account for the retarded acquisition of inhibitory responding as a result of CS preexposure. In this case, both CS and US representations are (primarily) in the A2 state during inhibition training trials, thereby retarding the CS from entering into an inhibitory relationship with the US. However, such an account cannot explain how the presence of CS A during the inhibition trials of Phase 2 abolishes the preexposure effect (i.e., allows CS X to become inhibitory). As the model is currently formulated, there is no reason why CS A would prevent the context from priming CS X into the A2 state, especially considering that such priming occurs prior to the presentation of the AX compound (i.e., the onset of the context occurs when the subjects are placed into the conditioning chamber, whereas the onset of the AX compound occurs many minutes later, allowing the context plenty of time to acti- 
vate most of the representation of CS X into the $\mathrm{A} 2$ state). Thus, in the framework of SOP, the presence of CS A should not protect CS X from the preexposure effect.

Dickinson and Burke (1996), however, proposed a modified version of SOP that can account for the attenuating effect of CS preexposure on the overshadowing of explicitly unpaired conditioned inhibition. Specifically, this revised version of SOP predicts that preexposure to target CS X will result in the formation of a context-X within-compound association. During explicitly unpaired inhibition training, this association should allow the context to activate a representation of $\mathrm{X}$ into state $\mathrm{A} 2$. As the US is intermittently presented during the inhibition training sessions, activation of the CS representation in state A2 at this time should facilitate the development of explicitly unpaired inhibition to $\mathrm{X}$, which is the same prediction that is made by the comparator hypothesis. (Note that although both models predict faster acquisition of explicitly unpaired inhibition following CS preexposure, neither model predicts a difference in the asymptote for this inhibition between groups that did and did not receive CS preexposure treatment. As we stated earlier, it is likely that this is the reason for our failure to observe different levels of inhibitory behavior in Groups CI.NoPre and CI.Pre.) Moreover, Dickinson and Burke anticipate that the presence of A during explicitly unpaired inhibition training (in the absence of CS preexposure) should produce overshadowing of the context $-\mathrm{X}$ association. However, their model predicts that the facilitation of explicitly unpaired inhibition by CS preexposure and the impairment of explicitly unpaired inhibition by overshadowing should summate. In contrast, the extended comparator hypothesis predicts that preexposure to $\mathrm{X}$ should go beyond summation and actively prevent overshadowing of $\mathrm{X}$, thereby creating an interaction with respect to the resultant inhibitory status of $X$ between overshadowing treatment and preexposure to $\mathrm{X}$. This anticipated interaction was in fact observed in both Experiment 1 and Experiment 2.

In contrast to these associative accounts, all of which are based on the acquisition of negative associative strength, the extended comparator hypothesis assumes that there are no negative associations and that behavior indicative of conditioned inhibition arises from select relationships among positive associations. As was stated above, the extended comparator hypothesis assumes that, for Group OV.Pre, massive preexposure to CS $\mathrm{X}$ in Phase 1 will build a strong $X$-context within-compound association, whereas, owing to the comparatively few overshadowing trials in Phase 2, a relatively weaker X-A within-compound association develops during overshadowing treatment. Congruent with the tenet of the extended comparator hypothesis that the candidate comparator stimulus with the strongest within-compound association with the target CS will act as the CS's comparator stimulus, the context should serve as the comparator stimulus for $\mathrm{CS} \mathrm{X}$, because of the stronger $\mathrm{X}$-context, relative to $\mathrm{X}-\mathrm{A}$, within-compound associa- tion. The context should be highly excitatory, because of the unsignaled USs administered during explicitly unpaired conditioned inhibition training. At test, the weak (or nonexistent) US representation activated directly by CS X is compared with the strong US representation activated indirectly by the excitatory context, resulting in behavior indicative of conditioned inhibition in response to CS X. Thus, the preexposure-overshadowing effect supports an extended comparator hypothesis view of comparator stimulus competition effects in both excitatory and inhibitory behavior. ${ }^{1}$

\section{REFERENCES}

Barnet, R. C., Grahame, N. J., \& Miller, R. R. (1993a). Local context and the comparator hypothesis. Animal Learning \& Behavior, 21, 1-13.

Barnet, R. C., Grahame, N. J., \& Miller, R. R. (1993b). Local time horizons in Pavlovian learning. Journal of Experimental Psychology: Animal Behavior Processes, 19, 215-230.

Blaisdell, A. P., Bristol, A. S., Gunther, L. M., \& Miller, R. R. (1998). Overshadowing and latent inhibition counteract each other: Support for the comparator hypothesis. Journal of Experimental Psychology: Animal Behavior Processes, 24, 335-351.

Blaisdell, A. P., Gunther, L. M., \& Miller, R. R. (1999). Recovery from blocking achieved by extinguishing the blocking CS. Animal Learning \& Behavior, 27, 63-76.

Bouton, M. E., \& Bolles, R. C. (1979). Contextual control of the extinction of conditioned fear. Learning \& Motivation, 10, 445-466.

Bouton, M. E., \& King, D. A. (1983). Contextual control of the extinction of conditioned fear: Tests for the associative value of the context. Journal of Experimental Psychology: Animal Behavior Processes, 9, 248-265.

Channel, S., \& Hall, G. (1983). Contextual effects in latent inhibition with an appetitive conditioning procedure. Animal Learning \& Behavior, 11, 67.74.

Cole, R. P., Barnet, R. C., \& Miller, R. R. (1995). Effect of relative stimulus validity: Learning or performance deficit? Journal of $E x$ perimental Psychology: Animal Behavior Processes, 21, 293-303.

Cole, R. P., Barnet, R. C., \& Miller, R. R. (1997). An evaluation of conditioned inhibition as defined by Rescorla's two-test strategy. Learning \& Motivation, 28, 323-341.

Denniston, J. C., Savastano, H. I., Blaisdell, A. P., \& Miller, R. R. (1999). Cue competition as a performance deficit. Manuscript submitted for publication.

Dickinson, A., \& BURKe, J. (1996). Within-compound associations mediate the retrospective revaluation of causality judgements. Quarterly Journal of Experimental Psychology, 49B, 60-80.

Friedman, B., Blaisdell, A. P., Escobar, M., \& Miller, R. R. (1998). Comparator mechanisms and conditioned inhibition: CS preexposure disrupts Pavlovian conditioned inhibition but not explicitly unpaired inhibition. Journal of Experimental Psychology: Animal Behavior Processes, 24, 453-466.

Grahame, N. J., Barnet, R. C., Gunther, L. M., \& Miller, R. R. (1994). Latent inhibition as a performance deficit resulting from CS-context associations. Animal Learning \& Behavior, 22, 395-408.

Grahame, N. J., Barnet, R. C., \& Miller, R. R. (1992). Pavlovian conditioning in multiple contexts: Competition between contexts for comparator status. Animal Learning \& Behavior, 20, 329-338.

HALL, G., \& MiNOR, H. (1984). A search for context-stimulus associations in latent inhibition. Quarterly Journal of Experimental Psychology, 36B, 145-169.

HeARST, E. (1972). Some persistent problems in the analysis of conditioned inhibition. In R. A. Boakes \& M. S. Halliday (Eds.), Inhibition and learning (pp. 5-39). London: Academic Press.

Kasprow, W. J., Catterson, D., Schachtman, T. R., \& Miller, R. R. (1984). Attenuation of latent inhibition by postacquisition reminder. Quarterly Journal of Experimental Psychology, 36B, 53-63. 
Kaufman, M. A., \& Bolles, R. C. (1981). A nonassociative aspect of overshadowing. Bulletin of the Psychonomic Society, 18, 318-320.

Kraemer, P. J., Hoffman, H., \& Spear, N. E. (1988). Attenuation of the CS-preexposure effect after a retention interval in preweanling rats. Animal Learning \& Behavior, 16, 185-190.

Kraemer, P. J., Randall, C. K., \& Carbary, T. J. (1991). Release from latent inhibition with delayed testing. Animal Learning \& Behavior, 19, 139-145.

Kraemer P. J., \& Roberts, W. A. (1984). The influence of flavor preexposure and test interval on conditioned taste aversions in the rat. Learning \& Motivation, 15, 259-278.

.uBow, R. E. (1973). Latent inhibition. Psychological Review, 79, 398 407.

_UBOW, R. E., \& MOORE, A. U. (1959). Latent inhibition: The effect of nonreinforced pre-exposure to the conditioned stimulus. Journal of Comparative \& Physiological Psychology, 52, 415-419.

_Ubow, R. E., Schnur, P., \& RIFKIN, B. (1976). Latent inhibition and conditioned attention theory. Journal of Experimental Psychology: Animal Behavior Processes, 2, 163-174.

Jubow, R. E., Weiner, I., \& Schnur, P. (1981). Conditioned attention theory. In G. H. Bower (Ed.), The psychology of learning and motivation (Vol. 15, pp. 1-49). New York: Academic Press.

IACKINTOSH, N. J. (1975). A theory of attention: Variations in the associability of stimuli with reinforcement. Psychological Review, $\mathbf{8 2}$ 276-298.

Aatzel, L. D., Schachtman, T. R., \& Miller, R. R. (1985). Recovery of an overshadowed association achieved by extinction of the overshadowing stimulus. Learning \& Motivation, 16, 398-412.

AATZel, L. D., Shuster, K., \& Miller, R. R. (1987). Covariation in conditioned response strength between stimuli trained in compound. Animal Learning \& Behavior, 15, 439-447.

Ailler, R. R., Barnet, R. C., \& Grahame, N. J. (1995). Assessment of the Rescorla-Wagner model. Psychological Bulletin, 117, 363386.

IILLER, R. R., Esposito, J. J., \& GrahamE, N. J. (1992). Overshadowinglike effects between potential comparator stimuli: Covariation in comparator roles of context and punctate excitor used in inhibitory training as a function of excitor salience. Learning \& Motivation, 23, $1-26$.

IILLER, R. R., \& MaTZEe, L. D. (1988). The comparator hypothesis: A response rule for the expression of associations. In G. H. Bower (Ed.), The psychology of learning and motivation (Vol. 22, pp. 5192). New York: Academic Press.

filler, R. R., \& SCHachtman, T. R. (1985). Conditioning context as an associative baseline: Implications for response generation and the nature of conditioned inhibition. In R. R. Miller \& N. E. Spear (Eds.), Information processing in animals: Conditioned inhibition (pp. 5188). Hillsdale, NJ: Erlbaum

APINI, M. R., \& BitTerman, M. E. ( 1993). The two-test strategy in the study of inhibitory conditioning. Journal of Experimental Psychology: Animal Behavior Processes, 19, 342-352.

AVLov, I. P. (1927). Conditioned reflexes (G. V. Anrep, Trans.). London: Oxford University Press.

EARCE, J. M. (1987). A model for stimulus generalization in Pavlovian conditioning. Psychological Review, 94, 61-73.

EARCE, J. M., \& HALL, G. (1980). A model for Pavlovian conditioning:
Variations in the effectiveness of conditioned but not unconditioned stimuli. Psychological Review, 87, 332-352.

REISS, S., \& WAGNER, A. R. (1972). CS habituation produces a "latent inhibition" effect but no active "conditioned inhibition." Learning \& Motivation, 3, 237-245.

ResCorla, R. A. (1971). Summation and retardation tests of latent inhibition. Journal of Comparative \& Physiological Psvchologv, 75, 77-81.

Rescorla, R. A., \& Wagner, A. R. (1972). A theory of Pavlovian conditioning: Variations in the effectiveness of reinforcement and nonreinforcement. In A. H. Black \& W. F. Prokasy (Eds.), Classical conditioning II: Current research and theory (pp. 64-99). New York: Appleton-Century-Crofts.

SUITER, R. D., \& LoLORDo, V. M. (1971). Blocking of inhibitory Pavlovian conditioning in the conditioned emotional response procedure. Journal of Comparative \& Physiological Psychology, 76, 137-144.

Van Hamme, L. J., \& Wasserman, E. A. (1994). Cue competition in causality judgments: The role of nonpresentation of compound stimulus elements. Learning \& Motivation, 25, 127-151.

WAGNER, A. R. (1981). SOP: A model of automatic memory processing in animal behavior. In N. E. Spear \& R. R. Miller (Eds.), Information processing in animals: Memory mechanisms (pp. 5-47). Hillsdale, NJ: Erlbaum.

Williams, D. A., LoLordo, V. M., \& Overmier, J. B. (1990). Signaling the unconditioned stimulus and backward conditioned inhibition: Prior conditioning of the signal and context. Learning \& Motivation, 21, 32-58.

\section{NOTE}

1. See Barnet et al., 1993b, Blaisdell et al., 1998, Friedman et al., 1998, Grahame et al., 1992, and Miller et al., 1992, for additional empirical evidence relating to how multiple nontarget stimuli compete for comparator status of the target CS. Also see Williams, LoLordo, and Overmier, 1990, for a similar finding involving backward inhibitory conditioning with a signaled (CS X) and unsignaled (CS Y) stimulus (i.e., $A \rightarrow U S \rightarrow X$ and $U S \rightarrow Y$, respectively). Pretraining the signal (A) with the US enhanced inhibition to the backward conditioned inhibitor that was signaled (CS X) but attenuated inhibition to the unsignaled backward inhibitor ( $\mathrm{CS} Y$ ). In the framework of the extended comparator hypothesis, CS X's comparator stimulus (CS A) was highly excitatory and, therefore, supported inhibition to CS X, whereas CS Y's comparator stimulus (the context) was modulated by its own comparator stimulus (CS A). It is likely that the highly excitatory CS A attenuated the magnitude of the indirect US representation activated by the context during testing on CS Y. This weaker indirect US was insufficient to support behavior indicative of inhibition in response to CS Y. Thus, the extended comparator hypothesis supplies a mechanism by which, as was suggested by Williams et al., "forward CS + preconditioning attenuated conditioning of the context and reduced the amount of backward conditioned inhibition on unsignaled trials" (p. 54).

(Manuscript received July 28, 1998: revision accepted for publication February 23, 1999.) 